ITEP-LAT/2002-23

KANAZAWA 02-32

\title{
The profile of the broken string in the confined and deconfined phase in full QCD *
}

\author{
V. Bornyakov ${ }^{\text {a }}$, H. Ichie ${ }^{\mathrm{b}}$, Y. Koma ${ }^{\mathrm{a}}$, Y. Mori ${ }^{\mathrm{a}}$, Y. Nakamura ${ }^{\mathrm{a}}$, M. Polikarpov $^{\mathrm{c}}$, G. Schierholz $^{\text {d }}$, \\ T. Streuer ${ }^{\mathrm{d}}$ and T. Suzuki ${ }^{\mathrm{a}}$ \\ - DESY-ITEP-Kanazawa Collaboration - \\ anstitute for Theoretical Physics, Kanazawa University, Kanazawa 920-1192, Japan \\ ${ }^{\mathrm{b}}$ Humboldt-Universität zu Berlin, Institut für Physik, D-10115 Berlin, Germany \\ ${ }^{\mathrm{c}}$ ITEP, B.Cheremushkinskaya 25, RU-117259 Moscow, Russia \\ dNIC/DESY Zeuthen, Platanenallee 6, D-15738 Zeuthen, Germany
}

\begin{abstract}
We study the profile of the broken string (flux tube) in the maximally abelian gauge below and above the finite temperature phase transition in full QCD. In the deconfinement phase, the flux tube disappears and the electric field apperas to be Coulomb-like. In the confinement phase, but near $T_{c}$, at shorter distances a flux tube is formed like at zero temperature, while at larger distances the tube disappears similar to the deconfinement phase.
\end{abstract}

\section{INTRODUCTION}

Regge trajectories and previous lattice QCD studies 11 suggest that the color electric field between quarks and antiquarks is squeezed into a one-dimensional flux tube (string). It can be interpreted by means of the dual superconductor picture of confinement as due to monopole condensation. Strong evidence for this scenario has been found in the abelian projected theory after fixing to the maximally abelian gauge [1].

At finite temperature the flux tube is expected to exist only in the low-temperature confinement phase and to disappear in the high-temperature deconfined phase. Lattice studies of the flux tube at finite temperature were done, for example, in quenched $\mathrm{SU}(2)$ in 2] and in quenched and full $\mathrm{SU}(3)$ in 3]. Flux tubes were found in the confinement phase, while no tubes have been observed in the deconfined phase. However, these authors did not study the behavior near the critical temperature in full QCD.

We shall study the flux tube near the critical temperature in full QCD. We denote the critical value of $\kappa$, at which the finite temperature phase transition takes place for a given value of $\beta$, by $\kappa_{t}$. (This should not be confused with the $\kappa$ value

\footnotetext{
${ }^{*}$ Talk given by H. Ichie
}

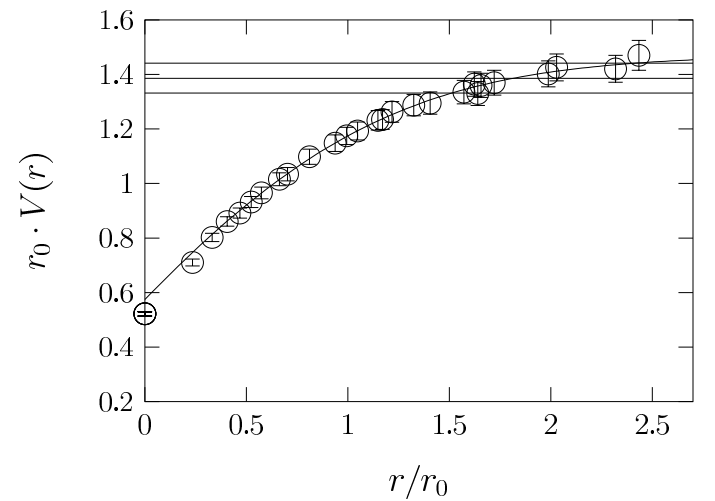

Figure 1. Static potential at $\beta=5.2$ and $\kappa=$ 0.1343 .

corresponding to the chiral limit.) As shown in Fig. 1 [4], in the confined phase slightly below $\kappa_{t}$ the quark potential rises linearly at shorter distances, but due to the presence of dynamical quarks it flattens at larger distances [5], as a result of string breaking.

\section{SIMULATION DETAILS}

We use non-perturbatively $O(a)$ improved Wilson fermions with $N_{f}=2$ flavours of dynamical quarks [4]. The simulations are done on $16^{3} 8$ lattices at $\beta=5.2$ for two $\kappa$ values, $\kappa=0.1360$ corresponding to the deconfined phase, and $\kappa=0.1343$ 

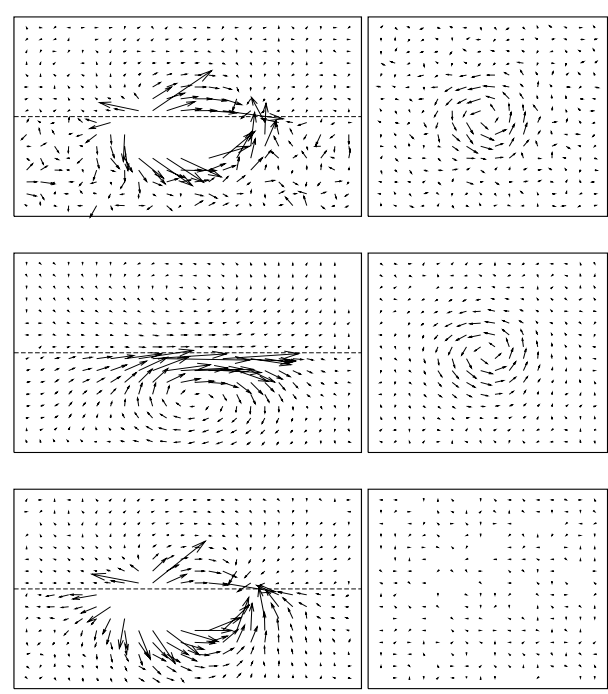

Figure 2. Color electric field (left) and monopole super current (right) in full QCD at zero temperature on the $16^{3} 32$ lattice after abelian projection (top), for the monopole contribution alone (middle), and the photon contribution (bottom). In the lower half of the left-hand plots the electric field is enhanced by a factor of 13, and arrows which are too large are omitted.

corresponding to the confinement phase. Our $\kappa$ values correspond to $m_{\pi} / m_{\rho}=0.4$ and 0.8 , respectively, at zero temperature [6]. The link variables are brought into the maximally abelian gauge using the simulated annealing algorithm of [7].

The abelian flux tube at finite temperature is probed by local observables, which are obtained from the correlation function of abelian Polyakov loops $L(x)$ and appropriate operators $O(y)$ [1] [], such as the color electric field and the monopole current:

$\frac{\left\langle O(y) L(0) L^{\dagger}(R)\right\rangle}{\left\langle L(0) L^{\dagger}(R)\right\rangle}-\langle O\rangle$.

\section{ABELIAN FLUX TUBE AT ZERO TEMPERATURE}

The basic idea of the dual superconductor picture of confinement is that the color electric field
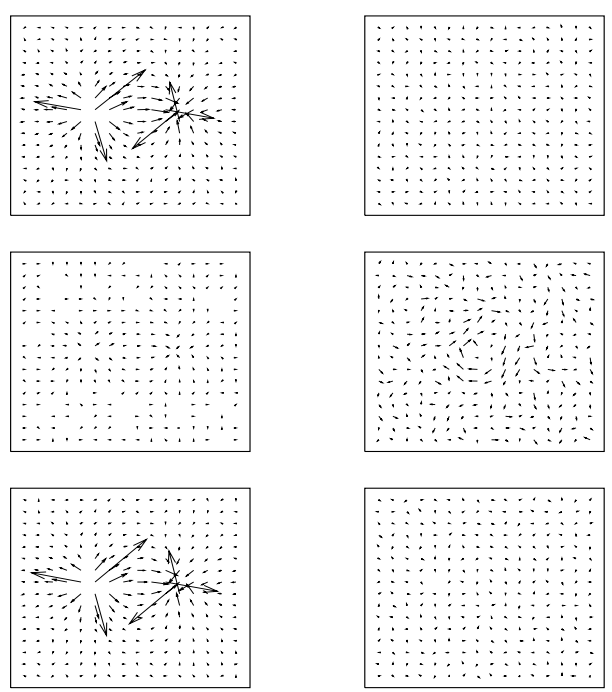

Figure 3. Color electric field (left) and monopole super current (right) for the total abelian field, the monopole and photon parts (from top to bottom) in the deconfined phase at $\kappa=0.1360$.

is squeezed into flux tubes by the monopole super current, which can be expressed by dual Ampère's law. In Fig. 2, which is borrowed from our zero temperature studies [7], we see that the abelian projected color electric field is indeed constricted to a tube. To elucidate the confinement mechanism a little further, we have decomposed the abelian field into its monopole part, which carries the monopole current, and the photon part, which carries the electric current [8]. Fig. 2 shows that when two external color electric charges of opposite sign are put into the QCD vacuum, the induced electric field shows up primarily in the photon field, while the monopole super current induces an electric field which exactly cancels the external field. As the signal from the monopole part of the field alone is much cleaner than that from the total abelian field, it sometimes helps to consider this part of the distribution only.

\section{ABELIAN FLUX TUBE AT FINITE TEMPERATURE}

In Figs. 3 and 4 we show the color electric field and the action density [7] in the deconfined phase. 

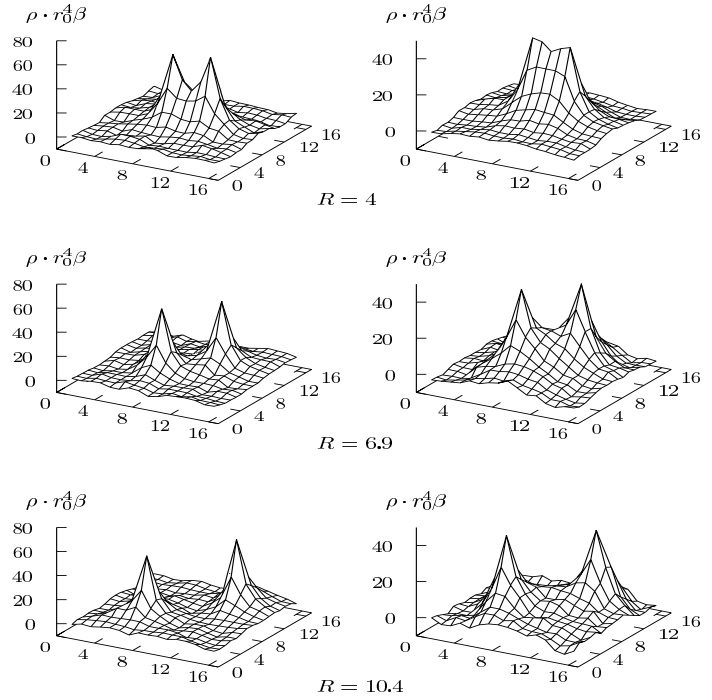

Figure 4. Action density in the deconfined phase at $\kappa=0.1360$ (left), and in the confinement phase at $\kappa=0.1343$ (right).

We see that the electric field is entirely given by the photon field, while the monopole part shows no (induced) color electric field. We observe no screening by dynamical quarks. The electric field looks Coulomb-like, like in the quenched case. In the action density we see only two peaks, while there is no signal of a flux tube at all interquark distances.

In Figs. 4 and 5 we show the action density and the color electric field in the confinement phase slightly below $\kappa_{t}$. We see a flux tube for smaller quark distances, which disappears for larger quark separations. A similar effect is seen in the monopole super current and the induced electric field (Fig. 5).

In conclusion, we have found evidence for the disappearance of the flux tube at large quark separations in the deconfined and the confinement phase due to the presence of dynamical quarks. This is consistent with the flattening of the static quark potential observed at large distances [- 1 .

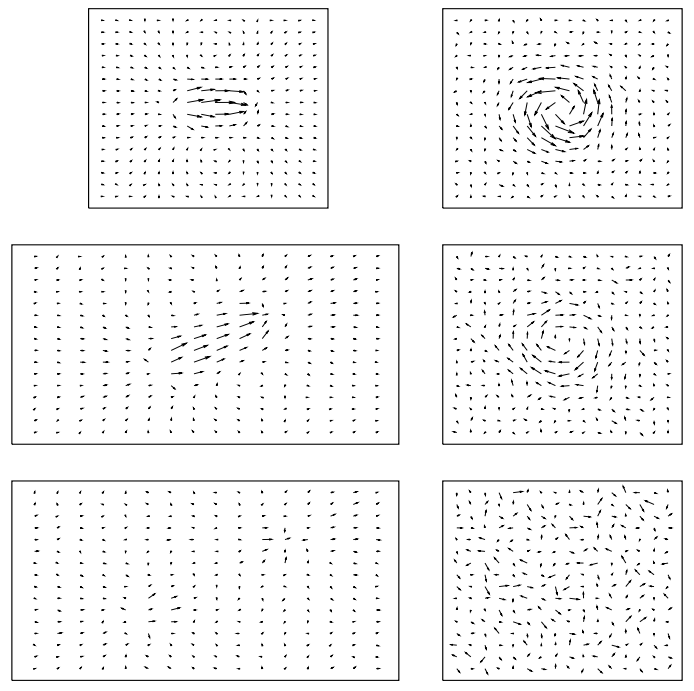

Figure 5. Color electric field (left) and monopole super current (right) for interquark distances of 0.5 (top), 0.8 (middle) and $1.3 \mathrm{fm}$ (bottom) from the monopole part of the abelian field in the confinement phase at $\kappa=0.1343$.

\section{ACKNOWLEDGEMENTS}

H. I. thanks the Humboldt university for hospitality. The numerical calculations have been done with COMPAQ AlphaServer ES40 at Humboldt university.

\section{REFERENCES}

1. K. Schilling et al., Nucl. Phys. B ( Proc. Suppl.) 73 (1999) 638.

2. Y. Peng and Y. Haymaker, Phys. Rev. D52 (1995) 3030.

3. W. Feilmair and H. Markum, Nucl. Phys. B370 (1992) 299.

4. V. Bornyakov et al., these proceedings heplat/0209157.

5. F. Karsch et al., Nucl. Phys. B605 (2001)579.

6. S. Booth et al. Phys. Lett. B519 (2001) 229.

7. V. Bornyakov et al. Nucl. Phys. B ( Proc. Suppl. ) 106 (2002) 634.

8. O. Miyamura Phys. Lett. B353 (1995) 91. 